\title{
Local Robust Performance Analysis for Nonlinear Dynamical Systems
}

\author{
Ufuk Topcu and Andrew Packard
}

\begin{abstract}
We propose a computational method for local robust performance analysis of nonlinear systems with polynomial dynamics. Specifically, we characterize upper bounds for local $\mathcal{L}_{2} \rightarrow \mathcal{L}_{2}$ input-output gains using polynomial Lyapunov/storage functions satisfying certain dissipation inequalities and compute safe approximations for these upper bounds via sum-of-squares programming problems. We consider both bounded parametric uncertainties and bounded uncertainties due to unmodeled dynamics.
\end{abstract}

\section{INTRODUCTION}

We consider the problem of quantifying robust performance properties of uncertain nonlinear dynamical systems with polynomial vector fields around asymptotically stable equilibrium points. The amount of amplification of bounded $\mathcal{L}_{2}$ input norms at the output channels is used as a measure of performance. Two types of uncertainties are considered: (1) bounded uncertainties due to unmodeled dynamics and (2) bounded parametric uncertainties. Following [1], [2], [3], we characterize upper bounds on local input-output gains due to bounded $\mathcal{L}_{2}$ disturbances by Lyapunov/storage functions which satisfy certain "local" dissipation inequalities [4]. Similar problems were studied in [1], [2], [5], [6], [7] mainly for systems with no uncertainty. Input-output properties of uncertain nonlinear systems were examined in [8] (for discrete time nonlinear systems with a finite-time horizon performance metric) and [9] (input-output gains for sufficiently small input signals).

In this paper, we use polynomial Lyapunov/storage function candidates, simple generalizations of the S-procedure [10], and sum-of-squares (SOS) relaxations for polynomial nonnegativity [11] and compute upper bounds on the inputoutput gains via (bilinear) SOS programming problems. Uncertainties due to unmodeled dynamics are accounted for in the setting [12] shown in Figure 1 where $M$ models the nominal part and $\Phi$ is an unknown operator satisfying certain relations between the input $z$ and the output $w_{2}$. The objective is to compute upper bounds on the $\mathcal{L}_{2}$ norm of the exogenous output $e$ in terms of the $\mathcal{L}_{2}$ norm of the exogenous input $w_{1}$. The approach is composed of two steps: first bound the $\mathcal{L}_{2}$ norm of the internal input $w_{2}$ to $M$ in terms of the $\mathcal{L}_{2}$ norm of $w_{1}$ and then perform an input-output gain analysis for $M$ from the inputs $\left(w_{1}, w_{2}\right)$ to the output $e$.

The approach for the bounded parametric uncertainties is similar to that developed in [13], [14] in the context of

U. Topcu is with Control and Dynamical Systems at California Institute of Technology, Pasadena, CA, 91125 (utopcu@cds. caltech .edu) and A. Packard is with the Department of Mechanical Engineering, The University of California, Berkeley, CA, 94720-1740, USA (packejagger.me.berkeley.edu).

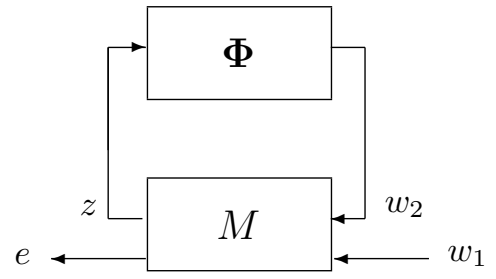

Fig. 1. Input-output system with the feedback interconnection of $\boldsymbol{\Phi}$ and M.

robust region-of-attraction analysis. Namely, a parameterindependent Lyapunov/storage function is used to characterize input-output properties of uncertain systems over the entire set of admissible values of uncertain parameters. The input-output relations characterized by a single parameterindependent certificate may be more conservative compared to those by parameter-dependent certificates. This potential conservatism is simply reduced by partitioning the set of uncertain parameters into subregions and computing parameterindependent certificates for each subregion. The partition of the uncertainty set can be refined following ideas parallel to branch-and-bound algorithm [15] to further reduce the conservatism. Although it is simplistic (compared to techniques based on parameter-dependent Lyapunov functions), this approach offers certain computational advantages as discussed in [14] for robust region-of-attraction analysis. In fact, in robustness analysis involving time-invariant unknown parameters, it is common, [16], [17], to combine easily-computable sufficient conditions with branch-and-bound strategies, often yielding improved analysis results.

The rest of the paper is organized as follows: A characterization of upper bounds for $\mathcal{L}_{2} \rightarrow \mathcal{L}_{2}$ input-output gains by Lyapunov/storage functions is discussed in section II. Section III is devoted to the development of the results for the case with unmodeled dynamics and this is followed by the method to account for parametric uncertainties in section IV. Implementation details are given in section V. Demonstration of the methodology with examples in section VI precedes the concluding remarks.

Notation: For $\xi \in \mathcal{R}^{n}, \xi \succeq 0$ means that $\xi_{k} \geq 0$ for $k=1, \cdots, n$. For $Q=Q^{T} \in \mathcal{R}^{n \times n}, Q \succeq 0(Q \succ 0)$ means that $\xi^{T} Q \xi \geq 0(>0)$ for all $\xi \in \mathcal{R}^{N}$. $\mathbb{R}[\xi]$ represents the set of polynomials in $\xi$ with real coefficients. The subset $\Sigma[\xi]:=$ $\left\{\pi=\pi_{1}^{2}+\pi_{2}^{2}+\cdots+\pi_{M}^{2}: \pi_{1}, \cdots, \pi_{M} \in \mathbb{R}[\xi]\right\}$ of $\mathbb{R}[\xi]$ is the set of SOS polynomials. For $\pi \in \mathbb{R}[\xi], \partial(\pi)$ denotes the degree of $\pi$. For $\eta>0$ and a function $g: \mathcal{R}^{n} \rightarrow \mathcal{R}$, define the $\eta$-sublevel set $\Omega_{g, \eta}$ of $g$ as

$$
\Omega_{g, \eta}:=\left\{x \in \mathcal{R}^{n}: g(x) \leq \eta\right\} .
$$


In several places, a relationship between an algebraic condition on some real variables and state properties of a dynamical system is claimed, and same symbol for a particular real variable in the algebraic statement as well as the state of the dynamical system is used. This could be a source of confusion, so care on the reader's part is required.

\section{UPPER BOUNDS ON THE $\mathcal{L}_{2} \rightarrow \mathcal{L}_{2}$ INPUT-OUTPUT GAIN}

Consider the dynamical system governed by

$$
\begin{aligned}
\dot{x}(t) & =f(x(t), w(t)) \\
z(t) & =h(x(t)),
\end{aligned}
$$

where $x(t) \in \mathcal{R}^{n}, w(t) \in \mathcal{R}^{n_{w}}$, and $f$ is a $n$-vector with elements in $\mathbb{R}[(x, w)]$ such that $f(0,0)=0$ and $h$ is an $n_{z}$-vector with elements in $\mathbb{R}[x]$ such that $h(0)=0$. Let $\phi\left(t ; \mathbf{x}_{0}, w\right)$ denote the solution to (1) at time $t$ with the initial condition $x(0)=\mathbf{x}_{0}$ driven by the input/disturbance $w$. For a piecewise continuous map $u:[0, \infty) \rightarrow \mathcal{R}^{m}$, define the (truncated) $\mathcal{L}_{2}$ norm as

$$
\|u\|_{2, T}:=\sqrt{\int_{0}^{T} u(t)^{T} u(t) d t .}
$$

For notational simplicity, denote $\|u\|_{2, \infty}$ by $\|u\|_{2}$.

\section{A. Upper bounds on local $\mathcal{L}_{2} \rightarrow \mathcal{L}_{2}$ gain}

Lemma II.1. [2] If there exist a real scalar $\gamma>0$ and $a$ continuously differentiable function $V$ such that, for $R>0$, $\Omega_{V, R^{2}}$ is bounded,

$V(0)=0$ and $V(x)>0 \quad$ for all nonzero $x \in \mathcal{R}^{n}$,

$\nabla V f(x, w) \leq w^{T} w-\gamma^{-2} z^{T} z \quad \forall x \in \Omega_{V, R^{2}}$ and $\forall w \in \mathcal{R}^{n_{w}}$,

then it holds that for the system in (1) and for all $T \geq 0$

$$
\|w\|_{2, T} \leq R \text { and } x(0)=0 \Rightarrow\|z\|_{2, T} \leq \gamma\|w\|_{2, T} .
$$

In other words, $\gamma$ is a local upper bound for the inputoutput gain for the system in (1). We call $\gamma$ to be a local upper bound because the upper bound on the norm of the output $z$ is only supposed to hold whenever the norm of the input is bounded by $R$. This is unlike the input-output gains for linear systems which hold for all values of input norms.

Let $\gamma>0$ be fixed and $\mathcal{V}$ be the space of continuously differentiable functions. Define $R_{\mathcal{L}_{2} \text {,opt }}(\mathcal{V}, \gamma)$ be the maximum value of $R$ such that the conditions in Lemma II.1 hold for some $V \in \mathcal{V}$. Let $\mathcal{V}_{\text {poly }}$ be a subset of $\mathcal{V}$ that is composed of all polynomials in $x$ of some fixed finite degree (omitted in notation). By restricting the search for $V$ satisfying the conditions in Lemma II.1 to $\mathcal{V}_{\text {poly }}$, utilizing a generalization of the S-procedure (see Lemma VIII.1 in the Appendix) to obtain sufficient conditions for the set containment constraints in Lemma II.1 and SOS relaxations for polynomial nonnegativity, the following proposition provides an upper bound on $R_{\mathcal{L}_{2} \text { opt }}(\mathcal{V}, \gamma)$.
Proposition II.1. For given $\gamma>0$ and positive definite polynomial l, let $R_{\mathcal{L}_{2}}$ be defined through

$$
\begin{gathered}
R_{\mathcal{L}_{2}}^{2}\left(\mathcal{V}_{\text {poly }}, \mathcal{S}, \gamma, l\right):=\max _{V \in \mathcal{V}_{\text {poly }}, R \geq 0, s \in \mathcal{S}} R^{2} \quad s . t . \\
V(0)=0, \quad s \in \Sigma[(x, w)] \\
V-l \in \Sigma[x] \\
-\left[\left(R^{2}-V\right) s+\nabla V f-w^{T} w+\gamma^{-2} z^{T} z\right] \\
\in \Sigma[(x, w)]
\end{gathered}
$$

where $\mathcal{V}_{\text {poly }} \subseteq \mathcal{V}$ is as defined above and $\mathcal{S}$ is a prescribed finite-dimensional subset of $\mathbb{R}[(x, w)]$. Then, $R_{\mathcal{L}_{2}}\left(\mathcal{V}_{\text {poly }}, \mathcal{S}, \gamma, l\right) \leq R_{\mathcal{L}_{2}, \text { opt }}(\mathcal{V}, \gamma)$

Note that $R_{\mathcal{L}_{2} \text {,opt }}$ depends on $\gamma$ and $\mathcal{V}$ and $R_{\mathcal{L}_{2}}$ depends on $\mathcal{V}_{\text {poly }}, \mathcal{S}, \epsilon, l$, and $\gamma$. Hereafter, this dependence will not be notated explicitly unless it causes confusion.

The optimization problem in Proposition II.1 can be cast in a bilinear SDP (i.e., nonconvex in general). Bilinear SDPs are known to be harder than linear ones [18]. Consequently, the state-of-the-art of the solvers for bilinear SDPs is far behind that for the linear ones and methods for bilinear SDPs are generally based on heuristics such as coordinate-wise affine search or specialized solvers e.g. PENBMI[19]. Although these techniques are local search schemes and convergence to a global optimum is not guaranteed, coupled with efficient initializations, they have been effectively used for several system analysis questions [20], [21]. For the examples in this paper, we use a coordinatewise affine search scheme as detailed in section $\mathrm{V}$.

For given $\gamma>0$, the optimization problem in Proposition II.1 maximizes $R$ (that can be verified through the families of admissible Lyapunov function candidates $(V)$ and S-procedure multipliers ( $s)$ ) such that $\|z\|_{2} \leq \gamma\|w\|_{2}$ whenever $\|w\|_{2} \leq R$. One can also choose to minimize $\gamma$ for a given value of $R$ and this can be formulated as an optimization problem similar to that in Proposition II.1 with minor changes.

\section{ROBUST PERFORMANCE IN THE PRESENCE OF UNMODELED DYNAMICS}

Consider the input-output system in Figure 1. Let

$$
\begin{aligned}
\dot{x}(t) & =f\left(x(t), w_{1}(t), w_{2}(t)\right) \\
z(t) & =h_{1}(x(t)) \\
e(t) & =h_{2}(x(t))
\end{aligned}
$$

be a realization of $M$, where $w_{1}(t) \in \mathcal{R}^{n_{w_{1}}}, w_{2}(t) \in \mathcal{R}^{n_{w_{2}}}$, $f$ is an $n$-vector of polynomials in $\mathbb{R}\left[\left(x, w_{1}, w_{2}\right)\right]$ with $f(0,0,0)=0, h_{1}$ and $h_{2}$ are $n_{z}$ and $n_{e}$ dimensional vectors with entries in $\mathbb{R}[x]$ satisfying $h_{1}(0)=0$ and $h_{2}(0)=0$. Furthermore, assume that $\boldsymbol{\Phi}$ is causal and, starting from rest, satisfies

$$
\|\mathbf{\Phi}(z)\|_{2, T}=\left\|w_{2}\right\|_{2, T} \leq\|z\|_{2, T}
$$

for all $T \geq 0$. Lemma III.1 provides a bound on $\left\|w_{2}\right\|_{2, T}$ in terms of $\left\|w_{1}\right\|_{2, T}$ for $T \geq 0$. In the following proposition, this result will be used to establish a local upper bound on the norm of the exogenous output $e$ in terms of the norm of the exogenous input $w_{1}$. 


$$
\begin{gathered}
R_{1}^{2}:=\max _{V \in \mathcal{V}_{\text {poly }}, R \geq 0, s \in \mathcal{S}} R^{2} \quad \text { subject to } \\
V(0)=0, \quad s \in \Sigma\left[\left(x, w_{1}, w_{2}\right)\right], \quad V-l \in \Sigma[x], \\
-\left[\left(R^{2}-V\right) s+\nabla V f\left(x, w_{1}, w_{2}\right)-\left(\beta^{2} w_{1}^{T} w_{1}+w_{2}^{T} w_{2}\right)+\alpha^{-2} z^{T} z\right] \in \Sigma\left[\left(x, w_{1}, w_{2}\right)\right], \\
\gamma_{1}:=\min _{Q \in \mathcal{V}_{\text {poly }}, \gamma>0, s \in \mathcal{S}} \quad \gamma \quad \text { subject to } \\
Q(0)=0, \quad s \in \Sigma\left[\left(x, w_{1}, w_{2}\right)\right], \quad Q-l \in \Sigma[x], \\
-\left[\left(\frac{R_{1}^{2}}{1-\alpha^{2}}-Q\right) s+\nabla Q f\left(x, w_{1}, w_{2}\right)-\left(\beta^{2} w_{1}^{T} w_{1}+w_{2}^{T} w_{2}\right)+\gamma^{-2} e^{T} e\right] \in \Sigma\left[\left(x, w_{1}, w_{2}\right)\right] .
\end{gathered}
$$

Lemma III.1. For $R>0,0<\alpha<1$ and $\beta>0$, if there exists a continuously differentiable, positive definite function $V$ such that $V(0)=0, \Omega_{V, R^{2}}$ is bounded, and

$$
\nabla V f\left(x, w_{1}, w_{2}\right) \leq \beta^{2} w_{1}^{T} w_{1}+w_{2}^{T} w_{2}-\frac{1}{\alpha^{2}} z^{T} z
$$

for all $x \in \Omega_{V, R^{2}}, w_{1} \in \mathcal{R}^{n_{w_{1}}}$, and $w_{2} \in \mathcal{R}^{n_{w_{2}}}$, then for $\boldsymbol{\Phi}$ starting from rest and for all $T \geq 0$

$$
x(0)=0 \text { and }\left\|w_{1}\right\|_{2, T} \leq \frac{R}{\beta} \Rightarrow\left\|w_{2}\right\|_{2, T} \leq \frac{\alpha R}{\sqrt{1-\alpha^{2}}} .
$$

Proof: While solutions to (14) exist, for $T \geq 0$

$$
\begin{aligned}
\beta^{2}\left\|w_{1}\right\|_{2, T}^{2}+\left\|w_{2}\right\|_{2, T}^{2}-\frac{1}{\alpha^{2}}\|z\|_{2, T}^{2} \\
\leq \beta^{2}\left\|w_{1}\right\|_{2, T}^{2}-\frac{1-\alpha^{2}}{\alpha^{2}}\|z\|_{2, T}^{2} \leq \beta^{2}\left\|w_{1}\right\|_{2, T}^{2} .
\end{aligned}
$$

Since $\Omega_{V, R^{2}}$ is bounded, as long as

$$
\left\|w_{1}\right\|_{2, T} \leq \frac{\sqrt{R^{2}-V(x(0))}}{\beta}
$$

solutions to (14) exist for all $T \geq 0$ and satisfy

$$
\left\|w_{2}\right\|_{2, T}^{2} \leq\|z\|_{2, T}^{2} \leq \frac{\alpha^{2} \beta^{2}}{1-\alpha^{2}}\left\|w_{1}\right\|_{2, T}^{2}+\frac{\alpha^{2}}{1-\alpha^{2}} V(x(0)) .
$$

In particular, for $x(0)=0, V(x(0))=$ and $\left\|w_{2}\right\|_{w, T} \leq$ $\frac{\alpha R}{\sqrt{1-\alpha^{2}}}$.

Proposition III.1. In addition to the conditions in Lemma III.1, if there exists a continuously differentiable, positive definite function $Q$ such that $Q(0)=0$ and

$$
\nabla Q f\left(x, w_{1}, w_{2}\right) \leq \beta^{2} w_{1}^{T} w_{1}+w_{2}^{T} w_{2}-\frac{1}{\gamma^{2}} e^{T} e
$$

for all $x \in \Omega_{Q, R^{2} /\left(1-\alpha^{2}\right)}, w_{1} \in \mathcal{R}^{n_{w_{1}}}, w_{2} \in \mathcal{R}^{n_{w_{2}}}$, then for $\Phi$ starting from rest and for all $T \geq 0$

$$
\left\|w_{1}\right\|_{2, T} \leq \frac{R}{\beta} \text { and } x(0)=0 \Rightarrow\|e\|_{2, T} \leq \gamma R / \sqrt{1-\alpha^{2}} .
$$

Proof: By Lemma III.1,

$$
\left\|w_{1}\right\|_{2, T} \leq R / \beta \Rightarrow \beta^{2}\left\|w_{1}\right\|_{2, T}^{2}+\left\|w_{2}\right\|_{2, T}^{2} \leq \frac{R^{2}}{1-\alpha^{2}} .
$$

Consequently, the result follows from Lemma II.1.
Lemma III.1 and Proposition III.1 can be used to construct relations between $\left\|w_{1}\right\|_{2, T}$ and $\|e\|_{2, T}$. Similar to Proposition II.1, one can obtain sufficient conditions for those in Lemma III.1 and Proposition III.1 using Lemma VIII.1 and SOS relaxations for polynomial nonnegativity. For given $\beta>0, \alpha>0$, and $l(x)=\epsilon x^{T} x$ ( with $\epsilon>0$ fixed), solve the problems in (8)-(10) and (11)-(13). Then, for $\boldsymbol{\Phi}$ starting from rest and for all $T \geq 0$

$$
x(0)=0 \text { and }\left\|w_{1}\right\|_{2, T} \leq \frac{R_{1}}{\beta} \Rightarrow\|e\|_{2, T} \leq \frac{\gamma R_{1}}{\sqrt{1-\alpha^{2}}} .
$$

When $\boldsymbol{\Phi}$ is unknown but a global gain relation between its inputs and outputs is known, then the results of this section provide a framework for robust performance analysis for the feedback interconnection between $M$ and $\boldsymbol{\Phi}$. On the other hand, even when the operator $\boldsymbol{\Phi}$ is known, the procedure outlined in this section can be used as a framework for compositional performance analysis. Note that conditions in Lemma III.1 and Proposition III.1 do not involve the states of (the realization of) $\Phi$. When $\Phi$ has the state space realization $\dot{x}_{2}(t)=f_{2}\left(x_{2}(t), z(t)\right)$, then the gain relation $\|\boldsymbol{\Phi}(z)\|_{2, T} \leq\|z\|_{2, T}$ can be established by determining a positive definite, continuously differentiable function $V_{2}$ satisfying

$\nabla V_{2} f_{2}\left(x_{2}, z\right) \leq z^{T} z-w_{2}^{T} w_{2} \quad \forall x_{2} \in \mathcal{R}^{n_{2}}, \quad \forall z \in \mathcal{R}^{n_{z}}$

which does not depend on the states $x_{1}$ of $M$. Consequently, Lemma III.1 and Proposition III.1 enable performance analysis for the feedback interconnection between $M$ and $\boldsymbol{\Phi}$ based on the input-output properties of individual blocks. Of course, this analysis may be conservative. Nevertheless, compositional analysis may be a fruitful direction which extends the applicability of SOS programming based nonlinear analysis tools for reasonably larger dimensional systems whenever it is possible to establish an interconnection structure as in Figure 1. Furthermore, it may be possible to refine the input-output relations between $w_{1}$ and $e$ by using transformations at the interconnections similar to the $D$ scales in linear robustness analysis [12]. 


\section{ROBUST PERFORMANCE ANALYSIS IN THE PRESENCE OF PARAMETRIC UNCERTAINTIES}

We now generalize the development in section II to the case where the vector field contains unknown but fixed and bounded parameters. Following the methodology proposed in [14] in the context of robust stability analysis, we first restrict our attention to

$$
\begin{aligned}
\dot{x}(t) & =f(x, w, \delta) \\
& :=f_{0}(x(t), w(t))+\sum_{i=1}^{m} \delta_{i} f_{i}(x(t), w(t)) \\
z(t) & =h(x(t)),
\end{aligned}
$$

where $f_{0}, f_{1}, \ldots, f_{m}$ are $n$-vectors with elements in $\mathbb{R}[(x, w)]$ such that $f_{0}(0,0, \delta)=f_{1}(0,0, \delta)=\ldots=$ $f_{m}(0,0, \delta)=0$, for all $\delta \in \boldsymbol{\Delta} \subset \mathcal{R}^{m}$, and $\boldsymbol{\Delta}$ is a known bounded polytope. Let $\phi\left(t ; \mathbf{x}_{0}, w, \delta\right)$ denote the solution of (17) for $\delta$ at time $t$ with the initial condition $x(0)=\mathbf{x}_{0}$ driven by the input/disturbance $w$ and $\mathcal{E}_{\boldsymbol{\Delta}}$ denote the set of vertices of $\Delta$

Proposition IV.1. If there exist a real scalar $\gamma>0$ and $a$ continuously differentiable function $V$ such that $V(0)=0$, $V(x)>0$ for all nonzero $x \in \mathcal{R}^{n}, \Omega_{V, R^{2}}$ is bounded, and

$$
\nabla V f(x, w, \delta) \leq w^{T} w-\gamma^{-2} z^{T} z
$$

for all $x \in \Omega_{V, R^{2}}, w \in \mathcal{R}^{n_{w}}$, and $\delta \in \mathcal{E}_{\Delta}$, then the system in (17) with $x(0)=0$ satisfies $\|z\|_{2} \leq \gamma\|w\|_{2}$ whenever $\|w\|_{2} \leq R$ and $\delta \in \boldsymbol{\Delta}$.

Proof: Since the vector field is affine in $\delta$ and $\boldsymbol{\Delta}$ is a bounded polytope, it follows that, for $\delta \in \boldsymbol{\Delta}$, $\nabla V f(x, w, \delta) \leq w^{T} w$ for all $x \in \Omega_{V, R^{2}}, w \in \mathcal{R}^{n_{w}}$. By Lemma II.1, for each $\delta \in \boldsymbol{\Delta},\|z\|_{2}=\|h(\phi(\cdot ; 0, w, \delta))\|_{2} \leq$ $\gamma\|w\|_{2}$ whenever $\|w\|_{2} \leq R$.

Note that restricting the attention to affine uncertainty $\delta$ dependence and polytopic $\boldsymbol{\Delta}$, Proposition IV.1 enables to compute upper bounds on $\mathcal{L}_{2} \rightarrow \mathcal{L}_{2}$ gain for the system in (17) by imposing the conditions in (18) at finitely many $\delta \in \mathcal{E}_{\boldsymbol{\Delta}}$ instead of at infinitely many $\delta \in \boldsymbol{\Delta}$. Furthermore, sufficient conditions for those in Proposition IV.1 can be obtained using Lemma VIII.1 and SOS relaxations.

The approach proposed here is restrictive: (1) only affine dependence on $\delta$ and polytopic $\boldsymbol{\Delta}$ are allowed (2) SOS relaxations for the conditions in Proposition IV.1 may include a large number of SDP constraints (3) single ( $\delta$-independent) Lyapunov/storage function is to certify properties for an entire family of systems. These limitations can be partially alleviated using techniques proposed in [14] in the context of robust region-of-attraction analysis. For example, polynomial dependence on $\delta$ in the vector field and the output map can be handled by covering the graph of non-affine functions $\delta$ (in the conditions in Proposition IV.1) by bounded polytopes. Furthermore, the fact that constraints in the SOS relaxations for the conditions in IV.1 are only coupled through the Lyapunov/storage functions (which include relatively small portion of all decision variables in associated SDPs) can be exploited through a suboptimal two-step procedure: pick a point in $\boldsymbol{\Delta}$, compute a Lyapunov/storage function for the system corresponding to that point, and then in the second step determine an input-output relation certified by the Lyapunov/storage function determined (fixed) in the first step which holds for the entire family of admissible systems. This procedure effectively decouples the large number of constraints in the second step enabling use of trivial parallelization. Finally, conservatism (due to using a single parameter-independent Lyapunov function and due to the suboptimal two-step procedure) can be reduced by an informal branch-and-bound type refinement procedure where $\Delta$ is partitioned into smaller subregions and a different Lyapunov/storage function is computed for each subregion. See [14] which develops a similar methodology in the context of robust region-of-attraction analysis.

\section{IMPLEMENTATION ISSUES}

The SOS relaxations in (5)-(7) lead to bilinear SDPs due to the multiplication between the decision variables in $V$ and the multipliers. Therefore, solution techniques for these problems are usually limited to local search schemes such as PENBMI [19] or coordinate-wise affine search based on the observation that, for given $V$ and $R$, constraints in these problems are affine in the decision variables in the multipliers. For example, one can obtain a suboptimal solution for the problem in (5)-(7) by alternatingly solving the following two problems until a maximum number of iterations is reacaed or the increase in the value of certified $R$ becomes smaller than a pre-specified tolerance . For given $V$

$$
\max _{R>0, s \in \mathcal{S}} R^{2} \text { subject to } s \in \Sigma[(x, w)] \text { and }(7),
$$

which can be solved using an off-the-shelf affine SDP solver through a line search on $R$, and for given (feasible) multiplier $s$

$\max _{R>0, V \in \mathcal{V}_{\text {poly }}} R^{2}$ subject to $V-l \in \Sigma[x], V(0)=0$, and $(7)$.

Furthermore, by a change of variables, it is possible to iterate without a line search in the first step. Indeed, for $\beta>0$, if the problem in (5)-(7) has the solution $R_{l}^{2}, V_{l}$ and $s_{l}$, then

$$
\begin{gathered}
\frac{1}{R_{l}^{2}}=\min _{K \in \mathcal{V}_{\text {poly }}, 1 / R^{2}>0, \tilde{s} \in \mathcal{S}} \frac{1}{R^{2}} \text { subject to } \\
\tilde{s} \in \Sigma[(x, w)], K \in \mathbb{R}[x], K-l_{1} / R^{2} \in \Sigma[x], \\
-\left[(1-K) \tilde{s}+\nabla K f-\frac{1}{R^{2}}\left(w^{T} w-\gamma^{-2} z^{T} z\right)\right] \\
\in \Sigma[(x, w)] .
\end{gathered}
$$

Note that for given $K$ constraints in (21) are affine in $1 / R^{2}$ and $\tilde{s}$. In fact, optimal values of $\tilde{s}$ and $K$ are $\tilde{s}=R_{l}^{2} s_{l}$, and $K=V_{l} / R_{l}^{2}$.

\section{EXAMPLES}

Consider the controlled short period aircraft dynamics in Figure 2 where $x_{p}:=\left[\begin{array}{lll}x_{1} & x_{2} & x_{3}\end{array}\right]^{T}, x_{1}, x_{2}$, and $x_{3}$ denote the pitch rate, the angle of attack, and the pitch angle, 


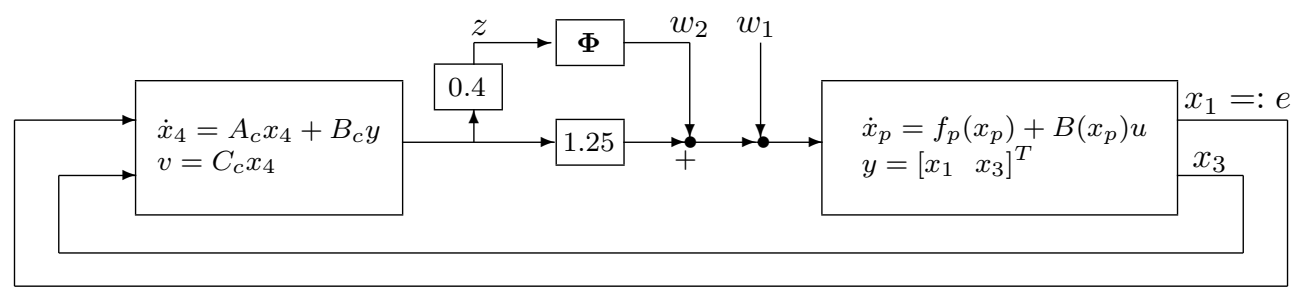

Fig. 2. Controlled short period aircraft dynamics with unmodeled dynamics.

respectively, and

$$
\dot{x}_{p}=\left[\begin{array}{c}
c\left(x_{p}\right) \\
q\left(x_{p}\right) \\
x_{1}
\end{array}\right]+\left[\begin{array}{c}
\ell_{b}^{T} x_{p}+b_{1} \\
b_{2} \\
0
\end{array}\right] u,
$$

where, $c$ and $q$ are cubic and quadratic polynomials, respectively, $\ell_{b} \in \mathcal{R}^{3}, b_{1}$ and $b_{2}$ are real scalars (see [22] for the values of the missing parameters). The plant output is $\left[\begin{array}{ll}x_{1} & x_{3}\end{array}\right]^{T}$. The input $u$ to the plant is

$$
u=1.25 v+w_{1}+w_{2}
$$

where $v$, the elevator deflection, is the controller output determined by

$$
\begin{aligned}
& \dot{x}_{4}=-0.864 y_{1}-0.321 y_{2} \\
& v=2 x_{4},
\end{aligned}
$$

where $x_{4}$ is the controller state. Assume that $\Phi: \mathcal{R} \rightarrow \mathcal{R}$ satisfies, starting from rest,

$$
\|\mathbf{\Phi}(z)\|_{2, T}=\left\|w_{2}\right\|_{2, T} \leq\|z\|_{2, T}
$$

for all $T \geq 0$. We performed the following analysis:

(i) For several values of $\alpha \in[0.55,0.9]$, solve the problems in (8)-(10) and (11)-(13).

(ii) Apply linearized robust performance analysis for the feedback interconnection [23] and fit a first order stable minimum phase transfer function, say $H(s)$, to the optimal D-scales. For several values of $\alpha \in[0.55,0.9]$, solve the problems in (8)-(10) and (11)-(13) for the system $H M H^{-1}$ with a minimal realization for $H$.

(iii) Solve the problem in Proposition II.1 for the system with no uncertainty for several values of $\gamma$.

Figure 3 shows the $\mathcal{L}_{2}$ norms of the exogenous outputs $e$ versus the $\mathcal{L}_{2}$ norms of the exogenous inputs $w_{1}$ in each of these cases: $(i)$ with marker "+", (ii) with marker "•", and (iii) with marker " $\times$ ".

Figure 3 illustrates the trade off between the robustness and performance: As $\alpha$ gets larger, the gap between the nominal performance level and the "robust" performance level increases deduced from the divergence between the curve with "+" and other two curves.

\section{CONCLUSION}

We proposed a computational method for local robust performance analysis of nonlinear systems with polynomial dynamics. Specifically, we characterized upper bounds for local $\mathcal{L}_{2} \rightarrow \mathcal{L}_{2}$ input-output gains using polynomial Lyapunov/storage functions satisfying certain dissipation inequalities and computed safe approximations for these upper bounds via sum-of-squares programming problems. We considered both bounded parametric uncertainties and bounded uncertainties due to unmodeled dynamics.

\section{Acknowledgements}

This work was sponsored by the Air Force Office of Scientific Research, USAF, under grant/contract number FA955005-1-0266. The views and conclusions contained herein are those of the authors and should not be interpreted as necessarily representing the official policies or endorsements, either expressed or implied, of the AFOSR or the U.S. Government.

U. Topcu acknowledges partial support from the Boeing Corporation. A. Packard acknowledges partial support from NASA under the contract NNX08AC80A.

\section{APPENDIX}

The following lemma is a simple generalization of the Sprocedure [10] and is used to obtain sufficient conditions for certain set containment constraints throughout the paper.

Lemma VIII.1. For $g_{0}, g_{1}, \cdots, g_{m} \in \mathbb{R}[x]$, if there exist $s_{1}, \cdots, s_{m} \in \Sigma[x]$ such that

$$
g_{0}-\sum_{i=1}^{m} s_{i} g_{i} \in \Sigma[x]
$$

then

$$
\begin{aligned}
\left\{x \in \mathcal{R}^{n}: g_{1}(x), \ldots, g_{m}(x)\right. & \geq 0\} \\
& \subseteq\left\{x \in \mathcal{R}^{n}: g_{0}(x) \geq 0\right\} .
\end{aligned}
$$

\section{REFERENCES}

[1] Z. Jarvis-Wloszek, R. Feeley, W. Tan, K. Sun, and A. Packard, "Control applications of sum of squares programming," in Positive Polynomials in Control, D. Henrion and A. Garulli, Eds. SpringerVerlag, 2005, pp. 3-22.

[2] W. Tan, A. Packard, and T. Wheeler, "Local gain analysis of nonlinear systems," in Proc. American Control Conf., Minneapolis, MN, 2006, pp. $92-96$.

[3] W. Tan, U. Topcu, P. Seiler, G. Balas, and A. Packard, "Simulationaided reachability and local gain analysis for nonlinear dynamical systems," in Proc. Conf. on Decision and Control, 2008.

[4] J. C. Willems, "Dissipative dynamical systems I: Genaral theory," Archive for Rational Mechanics and Analysis, vol. 45, pp. 321-343, 1972.

[5] S. Prajna, "Barrier certificates for nonlinear model validation," vol. 3, 2003, pp. 2884-2889. 


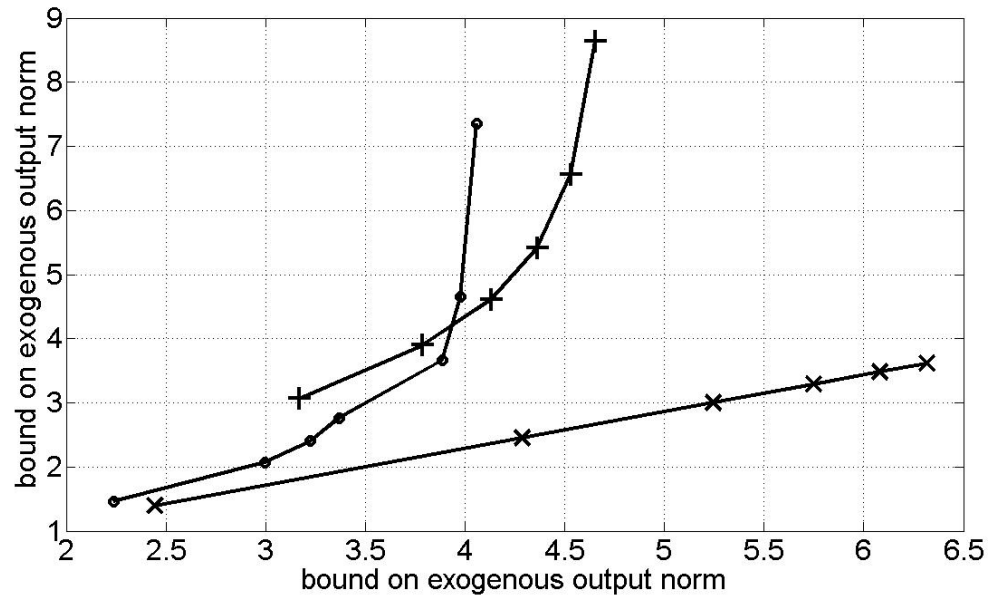

Fig. 3. The $\mathcal{L}_{2}$ norms of the exogenous outputs $e$ versus the $\mathcal{L}_{2}$ norms of the exogenous inputs $w_{1}$ in each of these cases: $(i)$ with marker "+", $(i i)$ with marker "•", and (iii) with marker " $\times$ ".

[6] D. F. Coutinho, M. Fu, A. Trofino, and P. Danes, " $\mathcal{L}_{2}$-gain analysis and control of uncertain nonlinear systems with bounded disturbance inputs," International Journal of Robust and Nonlinear Control, vol. 18, pp. 88-110, 2007.

[7] R. Vinter, "A characterization of the reachable set for nonlinear control systems," SIAM Journal on Control and Optimization, vol. 18, no. 6, pp. 599-610, 1980.

[8] J. Tierno and R. M. Murray, "Robust performance analysis for a class of uncertain nonlinear systems," in Proc. Conf. on Decision and Control, New Orleans, LA, 1995, pp. 1684-1689.

[9] H. Ito, "Local stability and performance robustness of nonlinear systems with structured uncertainty," IEEE Transactions on Automatic Control, vol. 44, no. 2, pp. 1250-1254, 1999.

[10] S. Boyd, L. E. Ghaoui, E. Feron, and V. Balakrishnan, Linear Matrix Inequalities in Systems and Control Theory. Philadelphia: SIAM, 1994.

[11] P. Parrilo, "Semidefinite programming relaxations for semialgebraic problems," Mathematical Programming Series B, vol. 96, no. 2, pp. 293-320, 2003.

[12] K. Zhou and J. C. Doyle, Essentials of Robust Control. Prentice Hall, 1998.

[13] U. Topcu and A. Packard, "Local stability analysis for uncertain nonlinear systems," 2007, to appear in IEEE Transaction on Automatic Control.

[14] U. Topcu, A. Packard, P. Seiler, and G. Balas, "Robust regionof-attraction estimation," 2008, submitted to IEEE Transaction on Automatica Control.

[15] E. Lawler and D.E.Wood, "Branch-and-bound methods: a survey," Operations Research, vol. 14, no. 4, pp. 679-719, 1966.

[16] V. Balakrishnan, S. Boyd, and S. Balemi., "Branch and bound algorithm for computing the minimum stability degree of parameterdependent linear systems," Int. J. of Robust and Nonlinear Control, vol. 1, no. 4, pp. 295-317, 1991.

[17] P. M. Young, M. P. Newlin, and J. C. Doyle, " $\mu$ analysis with real parametric uncertainty," in Proc. Conf. on Decision and Control, 1991, pp. 1251-1256.

[18] O. Toker and H. Ozbay, "On the NP-hardness of solving bilinear matrix inequalities and simultaneous stabilization with static output feedback," in Proc. American Control Conf., 1995, pp. 2525-2526.

[19] M. Kočvara and M. Stingl, "PENBMI User's Guide (Version 2.0), available from http://www. penopt.com," 2005.

[20] W. Tan and A. Packard, "Stability region analysis using sum of squares programming," in Proc. American Control Conf., Minneapolis, MN, 2006, pp. 2297-2302.

[21] U. Topcu, A. Packard, and P. Seiler, "Local stability analysis using simulations and sum-of-squares programming," Automatica, vol. 44, pp. $2669-2675,2008$

[22] U. Topcu, "Quantitative local analysis of nonlinear systems," Ph.D. Dissertation, UC, Berkeley, 2008, available at http://jagger.me.berkeley.edu/ utopcu/dissertation.
[23] G. Balas, J. Doyle, K. Glover, A. Packard, and R. Smith, " $\mu$-Analysis and Synthesis Toolbox - User's Guide," 1998. 